\title{
ARTICLES
}

\section{An explanation of the sheath instability}

\author{
B. P. Pandeya) and Subrata Roy \\ Computational Plasma Dynamics Laboratory, Kettering University, Flint, Michigan 48504
}

(Received 26 July 2002; accepted 9 October 2002)

When the sheath accelerates in its plasma-bound motion as a result of the ion response to the electric field, one has a top-heavy equilibrium. In the sheath frame ions are accelerated towards the wall-sheath boundary. In such a situation sheath may become unstable. The sheath instability is examined as the Rayleigh-Taylor (RT) instability and RT growth rate is compared with the reported sheath instability in the literature. (C) 2003 American Institute of Physics.

[DOI: $10.1063 / 1.1526091]$

\section{INTRODUCTION}

The sheath formation at the plasma-wall interface is ubiquitous in a bounded plasma. The specific feature of the plasma sheath is the formation of a charged boundary layer due to the difference in the mobility among different plasma particles, viz., electrons and ions in a two component plasma. Since electron mobility is much higher than the ion mobility, the plasma boundary becomes negatively charged leaving behind a positively charged column. The resultant potential gradient tends to slow incoming (to the boundary wall) electrons and accelerate the ions until a steady state is reached, with equal ion and electron fluxes. A stationary sheath exists only if the ion flow velocity satisfies the Bohm criteria at the plasma-sheath boundary, or if the electric field at the plasma-sheath interface exceeds some critical value. ${ }^{1-3}$ With no net current, the sheath width is typically few Debye length and the voltage drop, a few electron temperatures.

Consider an application where the boundary of the substrate is negatively biased, e.g., in plasma processing. Then, the static boundary sheath formation is limited to the case when fluctuations in the applied voltage at the negatively biased wall during the time of flight of ions through the sheath is negligible. If the fluctuation frequency in the applied voltage is smaller than the ion plasma frequency, the particles move in the quasistatic field of the sheath and static sheath model is a valid description. However, when fluctuation in the voltage and ion plasma frequency becomes comparable, the sheath field changes during the transition of the particle through the sheath, and static sheath description becomes invalid. ${ }^{4,5}$ The size and location of the plasma sheath may change due to the rapid variation at the plasma-sheath boundary and the sheath motion can be highly irregular and unstable. $^{6-12}$

When the sheath accelerates in its plasma bound motion as a result of the ion response to the electric field, one has a top-heavy equilibrium. In the sheath frame, plasma is accelerated towards the wall. This effective "gravity" pulls the

${ }^{a)}$ Electronic mail: bpandey@ kettering.edu heavy fluid through the plasma-sheath boundary layer as plasma density in the quasineutral region is more than near the negatively charged wall. The experimental observation of sheath instability in a double plasma device has been reported in the literature. ${ }^{12,13}$ Although experimental details of different sheath experiment varies, ${ }^{9-14}$ common features of observed sheath instability are the following: the oscillation frequency $f(\sim 100 \mathrm{kHz}-1 \mathrm{MHz})$ scales as the square root of the plasma number density in the source chamber, i.e, $f$ $\propto n_{s}^{1 / 2}$ and $f$ scales with the grid bias as $f \propto \phi^{-\alpha}$ with $\alpha$ between 0.5 and 0.25 . A different mechanism has been proposed to explain the sheath instability. For example, klystron bunching of the ions in the sheath, ${ }^{15}$ resonant interaction of the ions due to asymmetry in the sheath potential, ${ }^{11}$ potential relaxation instability ${ }^{9}$ or intermittent oscillation due to the onset of chaos ${ }^{16}$ are some of the possible mechanisms proposed to explain the instability.

In the present work, one develops a model whereby the sheath instability is explained in terms of Rayleigh-Taylor (RT) instability. The RT instability is believed to operate in space as well as in a laboratory environment. The instability has been extensively studied in a wide range of physical contexts both theoretically and experimentally. ${ }^{17-22}$ The primary source of the RT instability is the free energy available due to the presence of an inverted density gradient in the presence of gravitational force. In the vicinity of a near wall plasma region, number densities of plasma particles decline considerably. The electrons are repelled from the negatively biased wall whereas ions are accelerated towards the wall and thus their respective number density in the sheathpresheath region is much less than the corresponding number in the quasi-neutral presheath region. Therefore, the sheath region near the wall can be visualized as a "lighter fluid" under the "heavy fluid" of the quasi-neutral plasma region. The boundary of such an arrangement is accelerated towards the wall in the presence of an electric field. The resultant acceleration provides an effective "gravity." Thus, stationary sheath equilibrium is a "top-heavy" arrangement and a slight oscillation at the sheath-presheath boundary will help the 
heavy fluid to pierce through the lighter fluid. As a result, sheath instability may well be a manifestation of the Rayleigh-Taylor instability.

\section{BASIC MODEL}

We shall assume a collisionless sheath model and employ a fluid description to study the problem at hand. Due to the formation of sheath at the plasma boundary, there exist two regions in a bounded plasma: (a) The quasi-neutral bulk plasma, where electron and ion number densities $n_{e}$ and $n_{i}$ equal each other; and (b) the sheath at the boundary where $n_{e} \ll n_{i}$. We assume that the sheath-presheath boundary is located at $z=z_{0}$. The typical sheath width is a few Debye lengths (a spatial scale of a local electric field) that could be very small in practical applications, while the quasineutrality scale corresponds to the typical size of the system. This circumstance leads to nonuniversality of the plasma distribution functions for the whole region and allows the near wall sheath layer to be modeled separately from the bulk plasma region. ${ }^{2}$

Generally, governing equations of the sheath model are time independent. We shall consider such a sheathequilibrium near the wall. Most sheath models are based upon the assumption that electrons are in thermal equilibrium and may be given as $n_{e}=n_{0} \exp \left(e \phi / T_{e}\right)$, where $\phi$ is the sheath voltage near the wall and is always negative and $n_{0}$ is the plasma number density when $\phi=0$, and $T_{e}$ is the electron energy in $\mathrm{eV}$ with $e$ as the electronic charge. The steady state ion equation of motion is given as

$$
\begin{aligned}
& \boldsymbol{\nabla} \cdot\left(n_{i} \mathbf{v}_{\mathbf{i}}\right)=0, \\
& \boldsymbol{\nabla} \cdot\left[\left(m_{i} n_{i} \mathbf{v}_{\mathbf{i}}\right) \mathbf{v}_{\mathbf{i}}\right]=-\boldsymbol{\nabla} p_{i}+e n_{i} \mathbf{E}, \\
& \nabla^{2} \phi=4 \pi e n_{0}\left[\exp \left(\frac{e \phi}{T_{e}}\right)-\frac{n_{i}}{n_{0}}\right] .
\end{aligned}
$$

For the cold ion case,

$$
\frac{v_{i}^{2}}{2}+\frac{e \phi}{m_{i}}=C_{1} \text {. }
$$

Now making use of $n_{i} v_{i}=C_{2}$, one can write

$$
n_{i}=C_{2}\left[2\left(C_{1}-\frac{e \phi}{m_{i}}\right)\right]^{-1 / 2} \text {. }
$$

The boundary condition will require $C_{2}=n_{0} v_{i 0}$ and $C_{1}$ $=m_{i} v_{i 0}^{2} / 2$ as one must have $\phi(\infty)=0, n_{i}(\infty)=n_{0}$ and $v_{i}(\infty)=v_{i 0}$. The above set of equations reduces to a well known equation for the planner sheath,

$$
\nabla^{2} \phi=4 \pi e n_{0}\left[\exp \left(\frac{e \phi}{T_{e}}\right)-\left(1-\frac{2 e \phi}{m_{i} v_{i 0}^{2}}\right)^{-1 / 2}\right] .
$$

At the plasma-sheath boundary, $v_{i 0}=v_{\mathrm{B}}$, where $v_{\mathrm{B}}$ is the Bohm velocity. If sheath voltage is high, then Poisson's equation (3) can be simplified by assuming $n_{e}=0$ and

$$
\nabla^{2} \phi=-4 \pi e n_{i} .
$$

The above set of equations can be solved for a stationary sheath either with the zero potential at the plasma sheath boundary (the so called plasma model ${ }^{3}$ ) or with a nonzero electric field ${ }^{2}$ (the so called step sheath model). An analytical expression for plasm density, potential, etc. can be derived. The focus of the present work is to investigate the stability of a stationary sheath model and to that end, an applied electric field at the plasma-sheath boundary is assumed. The presence of such an electric field will accelerate the ions towards the wall and thus, the plasma-sheath layer can be modeled as a surface that separates the heavy fluid from the light fluid in the presence of an effective gravity. The inhomogeneity scale length based on the ambient density gradient for ion is $L_{n}^{-1}=n_{0}^{-1} d n_{0} / d z$. It is well known ${ }^{17}$ that the equilibrium with the inverted density gradient against gravity is unstable if $\mathbf{a} \cdot \nabla n_{0}<0$, where $\mathbf{a}$ is the acceleration. In order to study the instability of the sheath equilibrium, one perturbs the equilibrium quantities as $n_{i}(z)=n_{0}(z)+n_{1}(t, x, z), \quad \mathbf{v}_{i}=\mathbf{v}_{\mathbf{0}}(z)$ $+\mathbf{v}_{1}(t, x, z), p=p_{0}+p_{1}, \mathbf{E}=\mathbf{E}_{0}(z)+\mathbf{E}_{1}(t, x, z)$. Then the linearized equations are

$$
\begin{aligned}
& \frac{\partial n_{1}}{\partial t}+\boldsymbol{\nabla} \cdot\left(n_{0} \mathbf{v}_{1}+n_{1} \mathbf{v}_{0}\right)=0 \\
& m n_{0}\left[\frac{\partial \mathbf{v}_{\mathbf{1}}}{\partial t}+\mathbf{v}_{\mathbf{0}} \cdot \nabla \mathbf{v}_{1}+\mathbf{v}_{\mathbf{1}} \cdot \nabla \mathbf{v}_{0}\right]=-\nabla p_{1}+e n_{1} \mathbf{E}_{\mathbf{0}} .
\end{aligned}
$$

The perturbed electric field needs to be calculated selfconsistently using Poisson's equation. However, an imposed electric field at the plasma-sheath boundary ${ }^{2}$ is assumed here, i.e., $E_{z_{0}}=T_{e} / \lambda_{\mathrm{D}}$ where $\lambda_{\mathrm{D}}=\sqrt{T_{e} /\left(4 \pi n_{0} e^{2}\right)}$ is the Debye length defined at the plasma-sheath boundary.

The perturbation of the form $\exp [i(k x-\omega t)]$ is assumed. Then the linearized continuity equation (8) becomes

$$
-i \omega n_{1}+v_{1 z} n_{0}^{\prime}+v_{0} n_{1}^{\prime}=0
$$

where a prime denotes derivative $\partial / \partial z$. The components of Eq. (9) are given as

$$
\begin{aligned}
& m n_{0}\left(-i \omega v_{1 x}+v_{0} v_{1 x}^{\prime}\right)=-i k p_{1}, \\
& m n_{0}\left(-i \omega v_{1 z}+v_{0} v_{1 z}^{\prime}+v_{1 z} v_{0}^{\prime}\right)=-p_{1}^{\prime}+e n_{1} E_{0} .
\end{aligned}
$$

For simplicity, the incompressibility condition is assumed for the ion fluid, $\nabla \cdot \mathbf{v}_{i}=0$. Then,

$$
v_{x}=\frac{i}{k} \frac{\partial v_{1 z}}{\partial z} .
$$

After some simple algebra, from Eqs. (10)-(13) one gets

$$
\begin{aligned}
& \frac{\partial}{\partial z}\left[\frac{\partial v_{1 z}}{\partial z}-\frac{v_{0}}{\omega} \frac{\partial^{2} v_{1 z}}{\partial z^{2}}-\frac{i k^{2}}{\omega} v_{0} v_{1 z}\right] \\
& =k^{2}\left[1-\frac{\left(\frac{e}{m}\right) E_{0}\left(L_{n}^{-1}+\frac{n_{1}^{\prime}}{n_{0}}\right)}{\omega^{2}}+\frac{v_{0}^{\prime}}{i \omega}\right] v_{1 z} .
\end{aligned}
$$

Integrating the above equation from $-\epsilon$ to $+\epsilon$ across the plasma sheath boundary and invoking the continuity of velocity along with its derivative, one obtains

$$
\omega^{2}-\left(\frac{e}{m}\right) E_{0}\left(L_{n}^{-1}+\frac{n_{1}^{\prime}}{n_{0}}\right)-i \omega v_{0}^{\prime}=0 .
$$




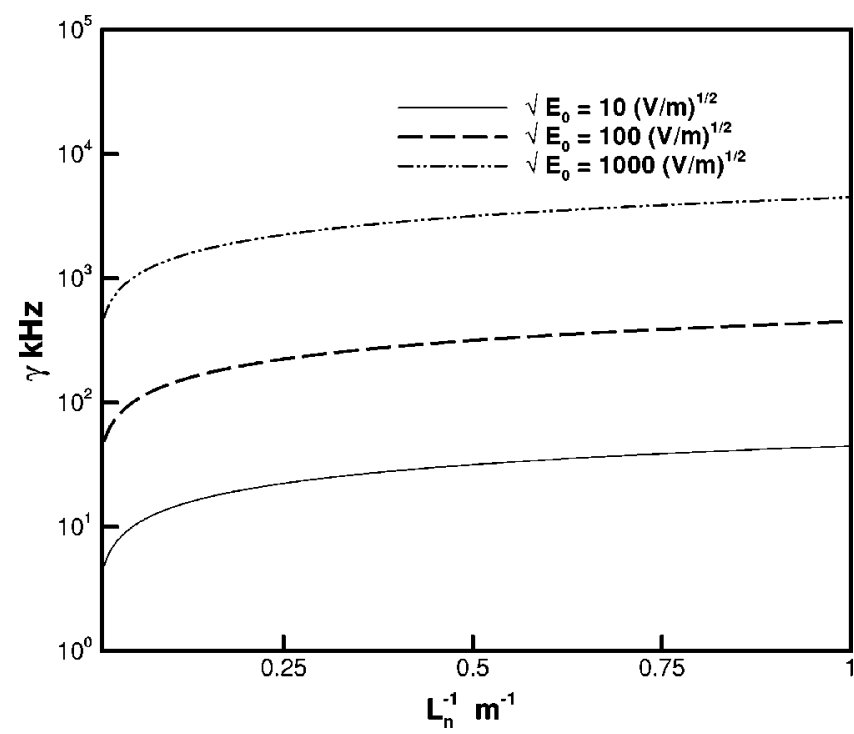

FIG. 1. The growth rate plotted against the inverse of inhomogeneity scale length for various values of the electric field shows that the linear growth frequency is about few $\mathrm{MHz}$ which compares favorably with the experiments (Refs. 11-15).

Before analyzing the above dispersion relation, one notes that $n_{1}^{\prime} / n_{0} \sim\left(v_{1 z} / v_{0}\right)\left(n_{0}^{\prime} / n_{0}\right) \ll L_{n}^{-1}$, and, thus, one may approximate $\left(L_{n}^{-1}+n_{1}^{\prime} / n_{0}\right)$ by $L_{n}^{-1}$. Assuming $\omega=\omega_{r}+i \gamma$, one finds from an imaginary part that

$$
\gamma=\frac{v_{0}^{\prime}}{2} \text {. }
$$

Equation (16) suggests that the plasma sheath boundary is unstable due the presence of the ion flow gradient. The free energy for the growth of this instability comes from the sheath potential as, for a cold ion case, flow velocity is related with the ambient potential ${ }^{23}$

$$
v_{0}^{2}=\frac{C_{s}^{2}}{2}\left[\exp \left(\frac{e \phi}{T_{e}}\right)-1\right],
$$

where ion acoustic speed $C_{s}^{2}=2 T_{e} / m_{i}$. In the absence of the flow, the above dispersion relation reduces to

$$
\omega^{2}=\frac{e E_{0}}{m L_{n}} .
$$

The growth rate is given as $\omega=\left(-e E_{0} / m L_{n}\right)^{0.5}$. The growth rate $\gamma$ can be written as

$$
\gamma=\frac{4.47 \times 10^{3}}{L_{n}^{0.5}} \sqrt{\frac{\Delta \phi}{\Delta z}},
$$

where $E_{0}=-\Delta \phi / \Delta z$. The sheath instability has been experimentally observed by many groups. ${ }^{7-15}$ In Fig. 1, the growth rate against the inverse of the scale length is plotted for different electric field values. As noted earlier, the growth rate is sensitive to the plasma density gradient scale and also to the applied field. The growth rate is high for a very sharp density gradient. Further, as the wall potential, and hence the corresponding sheath field increases, the growth rate also increases. The observed growth rate is between a few $\mathrm{KHz}$ to $\mathrm{Mhz}$ and a favorable comparison with our plotted number suggests that the RT mode might be the mechanism responsible for destabilizing the plasma-sheath boundary.

\section{CONCLUSIONS}

The plasma-sheath boundary in a plasma is a top-heavy arrangement with the inverted plasma density gradient against the "gravity." Gravity in this case is provided by the accelerating ion front. The inverted density gradient against the gravity is Rayleigh-Taylor unstable, and one demonstrates that the sheath instability can be explained as RT instability. The calculated growth rate matches reasonably well with the observed experimental data.

The transverse velocity shear can substantially reduce the growth rate of Rayleigh-Taylor instability in the short wave length regime. ${ }^{18,24}$ It is also known that the RT mode may self consistently generate a velocity shear which can then stabilize the mode. ${ }^{19}$ The nonlinear evolution of RT instability in the context of plasma sheath dynamics will be further explored in our future work.

In the case of a transient sheath, a situation germane to plasma processing, the plasma-sheath boundary initially propagates into the plasma at a velocity higher than the ion acoustic speed $C_{s}$ and at this time a refractive ion disturbance begins to propagate ahead of the sheath. ${ }^{25-27}$ Gradually its velocity drops below $C_{s}$. In the case of substantial ion-neutral collisions, the wave front does not get separated from the sheath. ${ }^{5}$ It is speculated that the supersonic sheath expansion and the subsequent subsonic sheath evolution in case of a transient sheath, might be triggered by the RT instability.

\section{ACKNOWLEDGMENTS}

Authors are grateful for the support of NASA Grants No. NAG3-2520 and No. NAG3-2638. One of the authors (S.R.) also wishes to acknowledge the support of the AFRL, Dayton, Ohio for the National Research Council fellowship (May-July, 2002) where part of his work was done.

${ }^{1}$ K. U. Riemann, J. Phys. D 24, 493 (1991).

${ }^{2}$ V. A. Godyak and N. Sternberg, Phys. Rev. A 42, 2299 (1990).

${ }^{3}$ N. Sternberg and V. A. Godyak, Physica D 97, 498 (1996).

${ }^{4}$ S. M. Malik, R. P. Fetheraston, K. Sridharan, and J. R. Conard, Plasma Sources Sci. Technol. 2, 81 (1993).

${ }^{5}$ M. J. Goeckner, S. M. Malik, J. R. Conard, and R. A. Breun, Phys. Plasmas 1, 1064 (1994).

${ }^{6}$ M. H. Cho, N. Hershkowitz, and T. Intrator, J. Vac. Sci. Technol. A 6, 2978 (1988).

${ }^{7}$ P. J. Barret and R. G. Greaves, Phys. Fluids B 1, 1776 (1989).

${ }^{8}$ M. Kono and A. Komori, Phys. Fluids B 4, 3569 (1992).

${ }^{9}$ G. Popa and R. Schrittwieser, Phys. Plasmas 1, 32 (1994).

${ }^{10}$ C. A. Capeau, G. Prasad, G. Bachet, and F. Doveil, Phys. Plasmas 3, 3331 (1996).

${ }^{11}$ A. Sarma, H. Bailung, and J. Chutia, Phys. Plasmas 3, 3245 (1996).

${ }^{12}$ A. Sarma, H. Bailung, and J. Chutia, Phys. Plasmas 4, 61 (1997).

${ }^{13}$ H. Klostermann, A. Rohde, and A. Piel, Phys. Plasmas 4, 2406 (1997).

${ }^{14}$ A. Piel, H. Klostermann, A. Rohde, N. Jelic, and R. Schtittwieser, Phys. Lett. A 216, 296 (1996).

${ }^{15}$ J. Chutia, S. Sato, H. Kubo, and Y. Nakamura, J. Plasma Phys. 46, 463 (1991).

${ }^{16}$ Y. Saitou, T. Honzawa, and T. Hada, Phys. Plasmas 2, 3605 (1995).

${ }^{17} \mathrm{~S}$. Chandrasekhar, Hydrodynamic and Hydromagnetic Stability (Dover, New York, 1961), p. 428. 
${ }^{18}$ P. Satyanarayana, P. Guzdar, J. D. Huba, and S. L. Ossakow, J. Geophys. Res. 89, 2945 (1984).

${ }^{19}$ J. Finn, Phys. Fluids B 5, 415 (1993).

${ }^{20}$ U. Shumlak and N. F. Roderick, Phys. Plasmas 5, 2384 (1998).

${ }^{21}$ B. Basu, Phys. Plasmas 5, 2022 (1998).

${ }^{22}$ B. vanderHolst, B. P. Pandey, and S. Poedts, Proceedings of the 6th International School/Symposia on Space Plasma Simulation, Garching, Germany, edited by J. Bchner, C. T. Dum, and M. Scholer (Copernicus, $\mathrm{GmbH}, 2001)$, p. 436.
${ }^{23}$ G. S. Kino and E. K. Shaw, Phys. Fluids 9, 567 (1966).

${ }^{24}$ P. N. Guzdar, P. Satyanarayana, J. D. Huba, and S. L. Ossakow, Geophys. Res. Lett. 9, 547 (1982).

${ }^{25}$ J. K. Chester, J. Sci. Technol. 37, 2 (1970).

${ }^{26} \mathrm{M}$. Widner, I. Alexeff, W. D. Jones, and K. E. Lonngren, Phys. Fluids 13, 2532 (1970).

${ }^{27}$ M. Shamim, J. T. Shurer, and J. R. Conard, J. Appl. Phys. 69, 2904 (1991). 